\title{
In-utero exposure to cigarette smoking on child long-term risk of obesity: concordance of self-report, maternal and cord blood biomarkers
}

\author{
Wenpin Hou ${ }^{1 *}$, Mingyu Zhang ${ }^{2,3}$, Yuelong $\mathrm{Ji}^{4}$, Xiumei Hong ${ }^{4}$, Guoying Wang ${ }^{4}$, Liming Liang ${ }^{5,6}$, Hongkai $\mathrm{Ji}^{1}$, Suchi \\ Saria ${ }^{7,8}$, Xiaobin Wang ${ }^{4,9 *}$.
}

1. Department of Biostatistics, Johns Hopkins University Bloomberg School of Public Health, Baltimore, MD

2. Department of Epidemiology, Johns Hopkins University Bloomberg School of Public Health, Baltimore, MD

3. Welch Center for Prevention, Epidemiology, and Clinical Research, Johns Hopkins University, Baltimore, MD

4. Center on the Early Life Origins of Disease, Department of Population, Family and Reproductive Health, Johns Hopkins University Bloomberg School of Public Health, Baltimore, MD

5. Department of Biostatistics, Harvard T.H. Chan School of Public Health, Boston, MA

6. Department of Epidemiology, Harvard T.H. Chan School of Public Health, Boston, MA

7. Department of Computer Science, Whiting School of Engineering, Johns Hopkins University, Baltimore, MD

8. Department of Health Policy and Management, Johns Hopkins University Bloomberg School of Public Health, Baltimore, MD

9. Department of Pediatrics, Johns Hopkins University School of Medicine, Baltimore, MD

* Correspondence: xwang82@jhu.edu (XW) and whou10@jhu.edu (WH).

\begin{abstract}
Most studies on the association of in utero exposure to cigarette smoking and childhood overweight or obesity (OWO) were based on maternal self-reported smoking data and few were based on objective biomarkers. In this study, we evaluated the associations between self-reported and biomarkers of in utero exposure to cigarette smoking with risk of childhood OWO. We analyzed data from 2351 mother-child pairs in the Boston Birth Cohort, a US low-income minority cohort that enrolled children at birth and followed prospectively up to age 18 years. In utero smoking exposure was measured by maternal self-report and by maternal and cord plasma cotinine and hydroxycotinine metabolites. We assessed the individual and joint associations of each smoking exposure measure and maternal OWO with childhood OWO using multinomial logistic regressions. We used nested logistic regressions to investigate the childhood OWO prediction performance when adding maternal and cord plasma biomarkers as input covariates on top of self-reported data. Our results demonstrated that in utero cigarette smoking exposure defined by self-report and by maternal or cord metabolites were consistently associated with increased risk of long-term child OWO. Adding maternal and cord plasma biomarker information to self-reported data improved the prediction accuracy of long-term child OWO risk.
\end{abstract}

Keywords: Maternal smoking; obesity; biomarkers; cotinine; hydroxycotinine; plasma; cord; in utero exposure

\section{Introduction}

The prevalence of child and adolescent obesity has been on the rise for the past four decades in the United States (US)1. Childhood obesity is associated with a myriad of adverse health consequences throughout lifetime, including higher risk of cardiovascular diseases, type 2 diabetes, certain cancers, and mental health issues ${ }^{2-6}$. In utero is a critical 
period when the fetus may be most vulnerable to environmental insults and susceptible to impacting multiple systems; such influence may extend into childhood and adulthood 7 . Multiple in utero environmental factors, such as endocrine disrupting chemicals ${ }^{8}$, ambient air pollutants ${ }^{9}$, toxic heavy metals ${ }^{10}$, and antibiotics ${ }^{11}$, have been linked with higher child body mass index (BMI) and/or higher risk of child overweight or obesity (OWO). Maternal cigarette smoking during pregnancy is another important and potentially modifiable environmental risk factor of child $\mathrm{OWO}^{12}$.

This study aims to address both methodological challenges and critical research questions related to in utero exposure to cigarette smoking. Methodologically, although the exposure-response association has been extensively examined ${ }^{13-15}$, most studies were based on self-reported cigarette smoking ${ }^{16-18}$. Characterizing in utero exposure to cigarette smoking based on self-report, however, is subject to potential recall bias, single-assessment bias, underreporting, and deliberate misreporting, all of which have been found to significantly introduce information bias ${ }^{19}$. In contrast, maternal biomarker measurements are objective. Also, since they are measured in mother's circulation ${ }^{20}$, it is more related to our goal of investigating to what extend they reflect fetal exposure, compared to other measurements such as cotinine levels in urine ${ }^{21,22}$. There is evidence that cotinine was detectable in fetal fluids in both active and passive smokers ${ }^{23}$. An average cigarette yields about $2 \mathrm{mg}$ of absorbed nicotine. Metabolized by cytochrome P450 enzymes and FMO3, nicotine converts to metabolites such as cotinine and 2-hydroxynicotine ${ }^{24}$. After cessation of smoking, cotinine can remain in the blood for up to 4 days ${ }^{25}$ and in the meantime be further metabolized as hydroxycotinine whose plasma elimination half-life time is $6.6 \mathrm{~h}^{26}$. However, most studies measured cotinine or its metabolites in maternal compartment, some others measured fetal fluids (amniotic fluid and blood) ${ }^{23}$. Few studies ${ }^{27}$ have simultaneously measured smoking biomarkers in both maternal and fetal compartment to evaluate their correlations and consistency with maternal self-reported data. Such data are particularly lacking in high-risk US minority populations. Cord biomarker measurements provide direct evidence of the fetal exposure, and prenatal exposure to smoking. Scientifically, it is necessary to clarify the concordance and utility of maternal vs. fetal blood biomarkers of cigarette smoking, given the growing number of birth cohort studies in the US and around the world and the availability of both maternal and cord blood samples. A related research question is which sources of samples (maternal vs. fetal) should be obtained and what types of biomarkers (cotinine vs. hydroxycotinine) should be measured to best reflect fetal in utero smoking exposure and to best estimate its long-term influence on child health outcomes.

Using the unique database of the Boston Birth Cohort (one of the largest and longest running high-risk US minority birth cohorts), this will be the first study that has all three measurements (maternal self-report, maternal plasma biomarkers, cord plasma biomarkers) analyzed simultaneously to characterize the in utero exposure to cigarette smoking. Specifically, we aimed to investigate the association of the three sources of measures of in utero exposure to cigarette smoking with child OWO from childhood to adolescence. Findings from this study open the door for further investigation into maternal and fetal metabolism of nicotine and transplacental passage of nicotine metabolites.

\section{Materials and Methods}

\subsection{Study population}

This study included participants in the Boston Birth Cohort (BBC) who were enrolled at birth and followed prospectively at the Boston medical center (BMC). A detailed description of the BBC can be found in our previous studies ${ }^{41,42}$. Mothers were invited to participate in the BBC study 24 to 72 hours after delivery. With signing written informed consent, mothers were interviewed by trained research staff using a standardized questionnaire. Six months after birth, enrolled children who still received pediatric primary or special care from the BMC were invited to further participate in the postnatal follow-up study from birth up to age 21 years old. This analysis included 2351 mother-child pairs 
who had data on self-reported smoking and child OWO data up to 18 years of age. A flowchart of participant selection process for the analysis is shown in Supplementary Figure 1. The study protocol for the baseline and follow-up studies were approved by the Institutional Review Boards of Boston University Medical Center and the Johns Hopkins Bloomberg School of Public Health. No identifying information or images was included in this manuscript.

\subsection{Exposure: in utero exposure to cigarette smoking}

We used three types of in utero cigarette smoking exposure assessment: maternal selfreported cigarette smoking, maternal plasma biomarkers (cotinine and hydroxycotinine metabolites), and cord plasma biomarkers (cotinine and hydroxycotinine metabolites). Maternal self-reported cigarette smoking was collected by the standardized questionnaire administered at enrollment. Trained staff collected non-fasting maternal blood samples within 24 to 72 hours after delivery and umbilical cord blood samples at birth. We measured the peak intensity of cotinine and hydroxycotinine in maternal and cord plasma using liquid chromatography-tandem mass spectrometry (LC-MS) techniques at the Broad Institute Metabolite Profiling Laboratory at Massachusetts Institute of Technology.

\subsection{Outcome: childhood overweight or obesity}

Trained medical staff measured child weight and height using the same clinical protocol and equipment at each well-child visit. Before the analyses, we cleaned child weight and height data by removing extreme values and outliers according to growth curve. We used the US national reference data and calculated child age- and sex-specific BMI percentiles $^{43}$ and defined child overweight as BMI $\geq 85$ th percentile and child obesity as BMI $\geq 95$ th percentile for gender ${ }^{44}$. We used the last visit as the end point of child overweight or obesity, since older age obesity is more likely to persist into later life; this is consistent with our previous publications.

\subsection{Covariates: maternal and child characteristics}

We collected covariates including maternal age at delivery, race/ethnicity, education, parity, pre-pregnancy weight and height, perceived stress during pregnancy, child age, gestational age, and birth weight from the standardized questionnaire administered at enrollment. We abstracted maternal gestational or preexisting diabetes from the electronic medical records. We collected infant breastfeeding data from the first two-year follow-up questionnaire. We calculated child birthweight for gestational age (BW-GA) and categorized BW-GA into small for gestational age (SGA) (BW-GA $<10^{\text {th }}$ percentile), appropriate for gestational age (AGA) (BW-GA between $10^{\text {th }}$ to $90^{\text {th }}$ percentile), and large for gestational age (LGA) (BW-GA $>90^{\text {th }}$ percentile). We grouped children into exclusively breastfeeding, exclusively formula feeding, or both breast and formula feeding. Gestational age was obtained either based on the first day of the last menstrual period, or early prenatal ultrasonographic results ${ }^{41}$. We calculated maternal pre-pregnancy BMI as pre-pregnancy weight (in $\mathrm{kg}$ ) divided by squared height (in $\mathrm{m}$ ), and further dichotomized into non-OWO $\left(\mathrm{BMI}<25 \mathrm{~kg} / \mathrm{m}^{2}\right)$ and $\mathrm{OWO}\left(\mathrm{BMI} \geq 25 \mathrm{~kg} / \mathrm{m}^{2}\right)$.

\subsection{Statistical analysis}

We first assessed the concordance of maternal self-reported smoking (3-categories: continuous smoking, non-smoking, quit smoking) vs. metabolite biomarkers of smoking (continuous) using the Analysis of Variance (ANOVA) F-test. We also examined the correlations of maternal and cord plasma cotinine and hydroxycotinine. We then used multinomial logistic regression models to examine the independent effect of in utero smoking exposure (self-reported, maternal blood biomarkers, cord blood biomarkers) on levels of child OWO (normal weight, overweight, obese). We modelled maternal and cord biomarkers (cotinine, hydroxycotinine, and their sum) as categorical variables (in quartiles) and as continuous variables (z-score). We used multinomial logistic regression models to 
examine the joint effects of in utero smoking exposure and maternal OWO on child OWO. We modelled maternal and cord biomarkers as binary variables (first to third quartile vs. fourth quartile). We examined the associations of cigarette biomarkers (continuous) and risk of childhood OWO (yes, no) by different levels of maternal OWO using a Generalized Additive Model (GAM). In this analysis, we considered values beyond -2 and 2 as outliers and excluded them in model fitting. We investigated the child OWO prediction performance when adding information of maternal and cord plasma biomarkers on top of selfreport smoking to the input covariates by three nested logistic regression models. The averaged AUROC from ten-fold cross-validation (train:test = 9:1) was used as the performance statistics.

In all regression analyses, we adjusted for confounders including maternal age at delivery, race/ethnicity, education level, parity, perceived stress during pregnancy, child age, gestational or preexisting diabetes, and breastfeeding. In a sensitivity analysis, we additionally adjusted for fetal growth restriction (SGA, AGA, LGA). We considered potential effect modifiers including child sex (male, female), preterm birth status (yes, no), and BW-GA (SGA, AGA, LGA). We conducted subgroup analyses by these potential effect modifiers and included interaction terms in the regression models to assess the statistical significance of these effect modifications.

We performed causal mediation test to examine whether the association of in utero exposure to cigarette smoking and child OWO was mediated by fetal growth restriction. For each comparison (childhood overweight or obesity, both were compared to non-OWO as a referent), probit regression models were fitted to regress SGA (yes or no) on maternal smoking, and to regress child OWO on SGA and smoking. We tested the significance of the indirect effect using the quasi-Bayesian Monte Carlo simulation with 1000 iterations. Unstandardized indirect effects were computed for each of 1000 Monte Carlo draws, and the $95 \%$ confidence interval was computed by determining the indirect effects at the $2.5^{\text {th }}$ and $97.5^{\text {th }}$ percentiles.

For all analyses, we considered $p$-value $<0.05$ as statistically significant. We used $\mathrm{R}$ packages mice (v.3.12.0) ${ }^{45}$, tableone (v.0.12.0) ${ }^{46}$, nnet (v.7.3.114) ${ }^{47}$, mediation (v.4.5.0 ${ }^{48}$, mgcv (v.1.8.31), PerformanceAnalytics (v.2.0.4) ${ }^{49}$, and ggplot (v.3.3.2) ${ }^{50}$ in this analysis. We imputed missing values using Multivariate Imputation by Chained Equations methods ${ }^{45}$. All methods were performed in accordance with the relevant guidelines and regulations.

\section{Results}

\subsection{Population Characteristics}

This study included 2351 mother-child pairs (Table 1-2). The prevalence of childhood overweight and obesity was $17.01 \%$ and $28.16 \%$, respectively. Childhood OWO was significantly associated with maternal age and pre-pregnancy BMI. Children with OWO had higher birthweight in measures including absolute weight in grams, standard birthweight in z-score, and birthweight for gestational age.

\subsection{Concordance of Self-Reported Smoking and Biomarkers}

The concordance of self-reported smoking and biomarkers was found in this data. Maternal self-reported cigarette smoking status was consistent with measures of maternal and cord plasma cotinine and hydroxycotinine, meaning the self-reported smoking data was reliable. In general, plasma cotinine, hydroxycotinine or their sum correlated well with maternal self-reported smoking status (Figure 1). Self-reported smoking status was significantly associated with cotinine, hydroxycotinine, and their sum: ANOVA F-test $p$ values were $4.94 \times 10^{-96}, 1.93 \times 10^{-109}, 1.75 \times 10^{-129}$ in maternal plasma, and $6.57 \times 10^{-73}, 4.04 \times 10^{-79}$, and $9.60 \times 10^{-91}$ in cord blood for cotinine, hydroxycotinine, and their sum, respectively. Concentrations of cotinine, hydroxycotinine, and their sum did not differ by maternal OWO status in either maternal or cord plasma (Supplementary Figure 2). The pairwise correlations among cotinine, hydroxycotinine, and their sum in 
maternal and cord plasma are shown in Supplementary Figure 3. Metabolites measured within maternal, or cord plasma were highly correlated; however, the correlations between maternal and cord metabolites measures were weak. This raised the question on which source of markers was more strongly associated with childhood OWO.

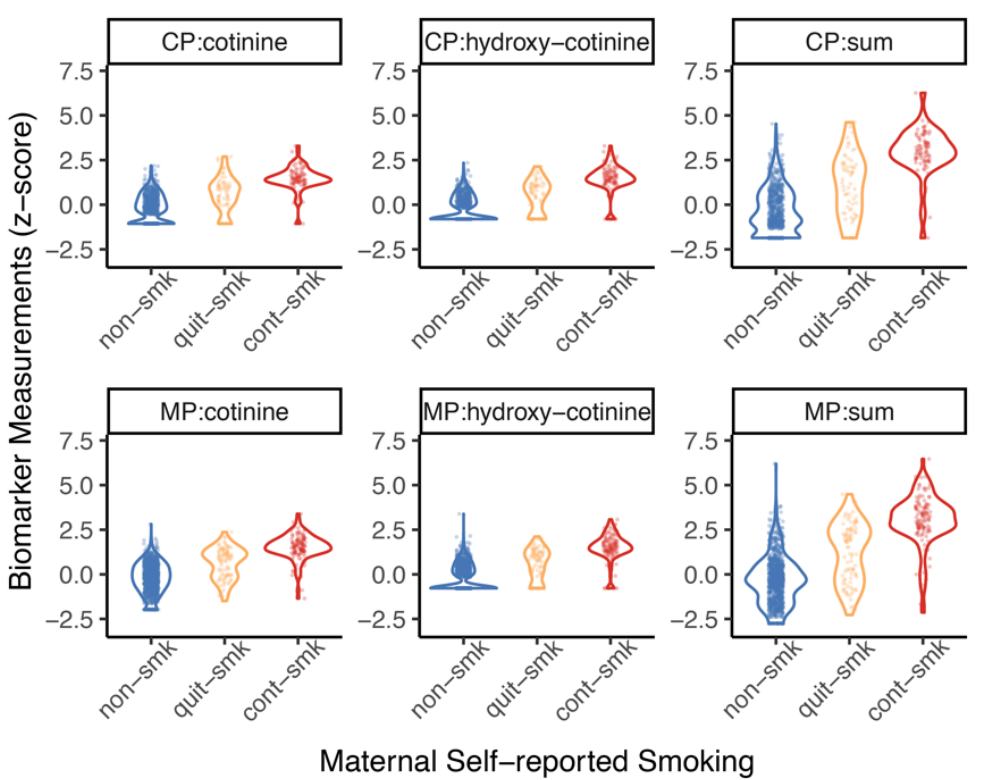

Figure 1. Continuous distribution of biomarker measures in maternal plasma (MP) and cord plasma (CP), stratified by maternal self-reported smoking status: non-smoking (non-smk), quitsmoking (quit-smk), and continuous-smoking (cont-smk). Dots show the measured values, and the violin plots show the distribution of these values (the width shows the probability density of the data at different values, smoothed by a kernel density estimator). The p-values obtained from ANOVA test in all the six measurements in the above subplots are $<2.2 \times 10^{-16}$. Notes: sum $=$ sum of cotinine and hydroxycotinine.

\subsection{Association of Self-Reported Cigarette Smoking and Childhood OWO}

Maternal self-reported smoking was not significantly associated with child OWO by itself, but its combined effect with maternal OWO enhanced children's long-term risk of OWO (Table 3). Such an association was additive with maternal OWO to enhance intergenerational link of maternal-childhood OWO. Compared to children born to non-OWO mothers who did not smoke, those born to non-OWO mothers who continued smoking had 1.73 (95\% CI: 1.01, 2.97) times the odds of having OWO, and those born to OWO mothers who did not smoke, quit smoking, and continued smoking had 2.98 (95\% CI: 2.73, 3.75), 3.63 (95\% CI: 2.32, 5.68), and 3.66 (95\% CI: 2.37, 5.67) times the odds of having OWO, after adjusting for potential confounders (Table 3). Stratification by child sex shows that this risk was stronger for overweight in female children and obesity in male children (Supplementary Table 2). Findings were consistent with the overall results when we stratified by preterm birth status (Supplementary Table 3) or fetal growth restriction (Supplementary Table 4).

\subsection{Association of Cigarette Smoke Biomarkers in Maternal Plasma and Childhood OWO}

As an individual covariate, children with Q4 level of cotinine had 1.37 (95\% CI: 0.87, 2.15) times the odds of being overweight and 1.51 (95\% CI: 1.03, 2.21) times the odds of being obese. Children OWO risk is highly differentiated between maternal OWO and maternal non-OWO groups (Figure 2). Compared to children of mothers who were nonOWO and had biomarker levels in Q1-Q3, the risk of overweight was higher for children whose mothers were OWO and were in Q1-Q3 levels of cotinine (OR=1.52, 95\% CI: 1.04, 2.21), hydroxycotinine $(\mathrm{OR}=1.69,95 \% \mathrm{CI}: 1.17,2.45)$, or their sum $(\mathrm{OR}=1.46,95 \% \mathrm{CI}: 1.00$, 2.12). This association was stronger for children of mothers with biomarkers levels in $Q 4$ : 
compared to those of mothers with non-OWO, children whose mothers were OWO had 2.44 (95\% CI: 1.47, 4.05), 1.59 (95\% CI: 0.95, 2.68), 2.40 (95\% CI: 1.47, 3.92) times the odds of being overweight for cotinine, hydroxycotinine, and their sum, respectively (Table 4). Obesity risk was significantly higher among children whose mothers were OWO (vs. nonOWO) and in Q1-Q3 levels of cotinine (OR = 2.63, 95\% CI: 1.92, 3.59), hydroxycotinine (OR $=3.02,95 \% \mathrm{CI}: 2.20,4.14)$, and their sum (OR = 2.96, 95\% CI: 2.15, 4.06); such risk was higher for children born to mothers who had Q4 level of cotinine (OR = 3.78, 95\% CI: 2.49, 5.75), hydroxycotinine ( $\mathrm{OR}=3.03,95 \% \mathrm{CI}: 2.00,4.59)$, and their sum $(\mathrm{OR}=3.34,95 \% \mathrm{CI}$ : $2.19,5.08)$ (Table 4). Children of whose mothers were OWO have higher odds of obesity from cotinine Q1-3 (OR = 2.63, 95\% CI: 1.92, 3.59) to Q4 (OR=3.78, 95\%CI: 2.49, 5.75), but the odds are comparable from hydroxycotinine Q1-3 (OR =3.02, 95\% CI:2.20, 4.14) to Q4 $(\mathrm{OR}=3.03,95 \% \mathrm{CI}: 2.00,4.59)$. In subgroup analyses, the combined effect of cotinine and maternal OWO was stronger for overweight in male children, and the cotinine by itself increased the male children's risk of obesity (Supplementary Table 5). The sex differences (generally stronger in female children but steeper increase in male children whose mothers were OWO) were also observed in the GAM that fitted childhood OWO risk versus biomarker measures (first-row plots in Supplementary Figure 3).

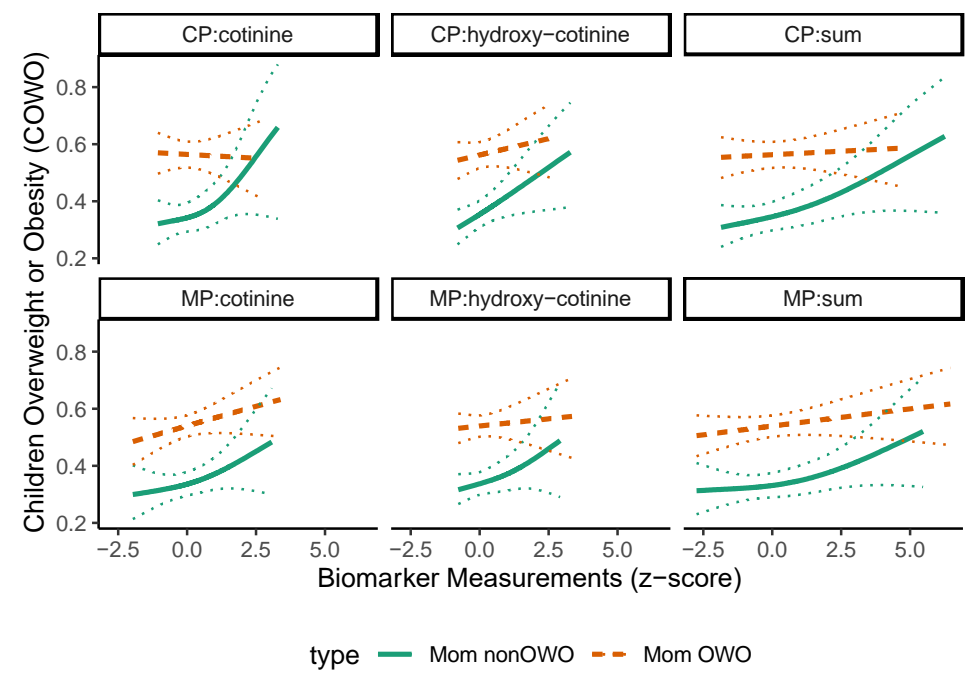

Figure 2. Assessment of child OWO risk by biomarkers of in-utero exposure to cigarette smoke, stratified by maternal OWO. Solid curves are GAM fitted values. Dotted curves denote 95\% CI. Note: MP, maternal plasma; $\mathrm{CP}$, cord plasma; sum, sum of cotinine and hydroxycotinine.

\subsection{Association of Cigarette Smoke Biomarkers in Cord Blood and Childhood OWO}

As an individual covariate, children with hydroxycotinine levels in Q4 had 1.66 (95\% CI: $1.03,2.66)$ times the odds of being overweight and $1.57(95 \% \mathrm{CI}: 1.05,2.36)$ times the odds of being obese, compared to those had hydroxycotinine levels in Q1 (Table 5). Children OWO risk is highly differentiated between maternal OWO and maternal non-OWO groups (Figure 2). The combined effect of maternal OWO and biomarkers (cotinine, hydroxycotinine) were significantly associated with both children overweight and obesity. For children born to non-OWO mothers, their risk of overweight was significantly higher for those with cord cotinine $(\mathrm{OR}=2.07,95 \% \mathrm{CI}: 1.09,3.94)$ and hydroxycotinine $(\mathrm{OR}=2.13$, 95\% CI: 1.13, 4.03) levels in Q4 (vs. Q1-3). Compared to children of non-OWO mothers and with cord hydroxycotinine levels in Q1-3, the odds of overweight were significantly increased in children of mothers with OWO and with cord hydroxycotinine levels in Q1$3(\mathrm{OR}=2.10,95 \% \mathrm{CI}: 1.36,3.26)$ and $\mathrm{Q} 4(\mathrm{OR}=2.57,95 \% \mathrm{CI}: 1.41,4.68)$. Risk of child obesity was also significantly higher with higher cord plasma cotinine levels, regardless of mothers OWO status. For children of non-OWO mothers, those with cord cotinine level in Q4 (vs. Q1-3) had 1.71 (95\% CI: 0.94, 3.10) times the odds of obesity. Results were consistent for hydroxycotinine $(\mathrm{OR}=1.52,95 \% \mathrm{CI}: 0.83,2.78)$ and their sum $(\mathrm{OR}=1.71,95 \% \mathrm{CI}$ : 0.96, 
3.06). Obese risk was higher in children of mother with $\mathrm{OWO}$ with odds significantly increased in cotinine from Q1-Q3 (OR $=2.97,95 \% \mathrm{CI}: 2.02,4.37)$ to Q4 (OR $=3.49,95 \% \mathrm{CI}$ : $2.10,5.79)$, hydroxycotinine from $\mathrm{Q} 1-3(\mathrm{OR}=2.79,95 \% \mathrm{CI}: 1.90,4.09)$ to $\mathrm{Q} 4(\mathrm{OR}=3.70,95 \%$ CI: $2.24,6.11)$, and their sum from $\mathrm{Q} 1-3(\mathrm{OR}=2.93,95 \% \mathrm{CI}: 1.99,4.31)$ to $\mathrm{Q} 4(\mathrm{OR}=3.74$, $95 \%$ CI: $2.25,6.23)$, all compared to children of mothers with non-OWO and the corresponding biomarker in Q1-Q3 (Table 5). This is different from the biomarker measurements in maternal plasma where such increasing trend of OR was not observed in hydroxycotinine (Table 4). Stratification by child sex showed that the cotinine's effect was mainly for obesity in male children and overweight in female children (Supplementary Table 6). The sex difference (generally higher risk in female children) was similar to what we observed in the maternal plasma (second-row plots in Supplementary Figure 4).

\subsection{Improved Prediction Performance When Adding Maternal and Cord Plasma Biomarkers}

The AUROC in the prediction of child non-OWO versus obesity (mean $=0.633$ ) was overall higher than that of child non-OWO versus overweight (mean $=0.583$ ). In the prediction model of child non-OWO versus obesity, the AUROC was 0.622 when including self-reported smoking as the smoking information (model 1); after adding maternal plasma biomarkers information (model 2), the AUROC increased to 0.637; and after adding cord plasma biomarkers information (model 3), AUROC increased to 0.638 (Supplementary Figure 5). The AUROC in the prediction of child non-OWO versus overweight also monotonically increased from model 1 to model 3 . This monotone increasing pattern of AUROC demonstrated the contribution of both maternal and cord plasma biomarkers in the prediction accuracy of child OWO.

\subsection{Mediation Analyses}

SGA mediated $47.3 \%$ of the total effect of maternal continuous smoking during pregnancy on child obesity. The regression coefficients between continuous smoking and child obesity and between SGA and child obesity were statistically significant $(\mathrm{p}=0.01$ and $1.75 \times 10^{-7}$, respectively). The average causal mediation effect (ACME) was -0.03 (95\% CI: $\left.-0.05,-0.02 ; \mathrm{p}<2 \times 10^{-16}\right)$. The average direct effect (ADE) was 0.10 (95\% CI: 0.03 , $0.18 ; \mathrm{p}=0.08)$. The total effect was 0.07 (95\% CI: $0.00,0.15, \mathrm{p}=0.05)$. The coefficient of smoking when regressed against SGA was $0.70\left(\mathrm{CI}: 0.47,0.93, \mathrm{p}=3.68 \times 10^{-9}\right)$ and the coefficient of SGA when regressed on child obesity was -0.55 (CI: $-0.76,-0.34, p=1.75 \times 10^{-7}$ ), suggesting that continuous smoking led to a higher chance of SGA, which further led to a decreased chance of obesity, resulting in a negative ACME. While in other comparison, the causal mediation effects were not statistically significant. In both sub-cohorts of 1315 mother-child pairs with maternal plasma metabolites and 893 mother-child pairs with cord plasma metabolites, birthweight for gestational age (a variable significantly associated with child $\mathrm{OWO}$ ) showed a significant interaction with non-smoking in children with obesity (Tables 4-5).

Table 1. Characteristics of participating mothers, overall and by maternal overweight or obese (OWO) status ${ }^{1}$.

\begin{tabular}{|l|c|c|c|c|c|}
\hline Variables & $\begin{array}{c}\text { Overall } \\
(\mathrm{N}=2351)\end{array}$ & $\begin{array}{c}\text { Non-OWO } \\
(\mathrm{N}=1289)\end{array}$ & $\begin{array}{c}\text { Overweight } \\
(\mathrm{N}=400)\end{array}$ & $\begin{array}{c}\text { Obesity } \\
(\mathrm{N}=662)\end{array}$ & $P$ \\
\hline Maternal Age (year) (\%) & $226(9.6)$ & $144(11.2)$ & $30(7.5)$ & $52(7.9)$ & \\
\cline { 1 - 5 }$>20$ & $1686(71.7)$ & $921(71.5)$ & $292(73.0)$ & $473(71.5)$ & 0.039 \\
\hline$>=20$ and $<35$ & $439(18.7)$ & $224(17.4)$ & $78(19.5)$ & $137(20.7)$ & \\
\hline$>=35$ & $1612(68.6)$ & $892(69.2)$ & $272(68.0)$ & $448(67.7)$ & 0.116 \\
\hline Race/Ethnicity (\%)
\end{tabular}




\begin{tabular}{|c|c|c|c|c|c|}
\hline Hispanic & 459 (19.5) & 228 (17.7) & $81(20.2)$ & $150(22.7)$ & \\
\hline Others & $153(6.5)$ & $92(7.1)$ & $26(6.5)$ & $35(5.3)$ & \\
\hline White & $127(5.4)$ & $77(6.0)$ & $21(5.2)$ & $29(4.4)$ & \\
\hline \multicolumn{6}{|l|}{ Education (\%) } \\
\hline Above college & $803(34.2)$ & $457(35.5)$ & $135(33.8)$ & $211(31.9)$ & \multirow{3}{*}{0.161} \\
\hline Below college & $1531(65.1)$ & $825(64.0)$ & $259(64.8)$ & $447(67.5)$ & \\
\hline Unknown & $17(0.7)$ & $7(0.5)$ & $6(1.5)$ & $4(0.6)$ & \\
\hline \multicolumn{6}{|l|}{ Parity (\%) } \\
\hline Multiparous & $1367(58.1)$ & $736(57.1)$ & $246(61.5)$ & $385(58.2)$ & \multirow{2}{*}{0.297} \\
\hline Nulliparous & $984(41.9)$ & $553(42.9)$ & $154(38.5)$ & $277(41.8)$ & \\
\hline \multicolumn{6}{|l|}{ Smoking (\%) } \\
\hline Continuous smoking & $233(9.9)$ & $117(9.1)$ & $42(10.5)$ & $74(11.2)$ & \multirow{3}{*}{0.296} \\
\hline Non smoking & $1930(82.1)$ & $1072(83.2)$ & $331(82.8)$ & $527(79.6)$ & \\
\hline Quit smoking & $188(8.0)$ & $100(7.8)$ & $27(6.8)$ & $61(9.2)$ & \\
\hline \multicolumn{6}{|c|}{ Perceived stress during pregnancy (\%) } \\
\hline High & $425(18.1)$ & $226(17.5)$ & $71(17.8)$ & $128(19.3)$ & \multirow{3}{*}{0.873} \\
\hline Low & $895(38.1)$ & $489(37.9)$ & $156(39.0)$ & $250(37.8)$ & \\
\hline Medium & $1031(43.9)$ & $574(44.5)$ & $173(43.2)$ & $284(42.9)$ & \\
\hline \multicolumn{6}{|c|}{ Pre-pregnancy BMI category (\%) } \\
\hline$<25$ & $1109(47.2)$ & $732(56.8)$ & $176(44.0)$ & $201(30.4)$ & \multirow{3}{*}{$<0.001$} \\
\hline $25-25.99$ & $662(28.2)$ & $350(27.2)$ & $120(30.0)$ & $192(29.0)$ & \\
\hline$>=30$ & $580(24.7)$ & $207(16.1)$ & $104(26.0)$ & $269(40.6)$ & \\
\hline \multicolumn{6}{|c|}{ Gestational or preexisting Diabetes } \\
\hline No & $2248(95.6)$ & $1244(96.5)$ & $379(94.8)$ & $625(94.4)$ & \multirow{2}{*}{0.065} \\
\hline Yes & $103(4.4)$ & $45(3.5)$ & $21(5.2)$ & $37(5.6)$ & \\
\hline
\end{tabular}

${ }_{1}^{1} \mathrm{OWO}$, overweight or obesity; SD, standard deviation; non-smoking, non-smoker (non-smoking smoke).

${ }^{2}$ nonOWO, overweight and obesity are defined as maternal $\mathrm{BMI}<25 \mathrm{~kg} / \mathrm{m} 2,25-29: 9 \mathrm{~kg} / \mathrm{m} 2$, and $\mathrm{BMI}>30 \mathrm{~kg} / \mathrm{m} 2$, respectively.

Table 2. Characteristics of participating children (paired with mothers in Table 1) $)^{1}$.

\begin{tabular}{|l|c|c|c|c|c|}
\hline Variables & $\begin{array}{c}\text { Overall } \\
(\mathrm{N}=2351)\end{array}$ & $\begin{array}{c}\text { Non-OWO2 } \\
(\mathrm{N}=1289)\end{array}$ & $\begin{array}{c}\text { Overweight } \\
(\mathrm{N}=400)\end{array}$ & $\begin{array}{c}\text { Obesity } \\
(\mathrm{N}=662)\end{array}$ & $P$ \\
\hline Child sex & $1174(49.9)$ & $651(50.5)$ & $195(48.8)$ & $328(49.5)$ & \multirow{2}{|l|}{0.806} \\
\hline \multicolumn{1}{|l|}{ Female } & $1177(50.1)$ & $638(49.5)$ & $205(51.2)$ & $334(50.5)$ & \\
\hline Children's Age (mean (SD)) & $10(4)$ & $9.37(4.02)$ & $9.90(4.02)$ & $9.97(3.87)$ & 0.003 \\
\hline Birth weight (mean (SD)) & 2920 & 2833.21 & 2981.36 & 3052.30 & \\
& $(818)$ & $(805.61)$ & $(805.08)$ & $(828.41)$ & $<0.001$ \\
\hline Standard birthweight (SBW) Z-score & $-0(1)$ & $-0.19(0.98)$ & $-0.05(1.04)$ & $0.18(1.06)$ & $<0.001$ \\
(mean (SD)) & $38(3)$ & $37.59(3.59)$ & $37.96(3.28)$ & $37.80(3.33)$ & 0.13 \\
\hline Gestational age (mean (SD)) & & & & \\
\hline Preterm birth & & & & & \\
\hline
\end{tabular}




\begin{tabular}{|c|c|c|c|c|c|}
\hline No & $1708(72.6)$ & $922(71.5)$ & $306(76.5)$ & $480(72.5)$ & \multirow{2}{*}{0.149} \\
\hline Yes & $643(27.4)$ & $367(28.5)$ & $94(23.5)$ & $182(27.5)$ & \\
\hline \multicolumn{6}{|c|}{ Birth weight for gestational age (\%) } \\
\hline Appropriate & $1829(77.8)$ & $1011(78.4)$ & $309(77.2)$ & $509(76.9)$ & \multirow{3}{*}{$<0.001$} \\
\hline Large & $228(9.7)$ & $84(6.5)$ & $38(9.5)$ & $106(16.0)$ & \\
\hline Small & $294(12.5)$ & $194(15.1)$ & $53(13.2)$ & $47(7.1)$ & \\
\hline \multicolumn{6}{|l|}{ Breastfeeding (\%) } \\
\hline Both & $1579(67.2)$ & $873(67.7)$ & $276(69.0)$ & $430(65.0)$ & \multirow{3}{*}{0.172} \\
\hline Bottle fed & $597(25.4)$ & $313(24.3)$ & $94(23.5)$ & $190(28.7)$ & \\
\hline Exclusive breastfed & $175(7.4)$ & $103(8.0)$ & $30(7.5)$ & $42(6.3)$ & \\
\hline
\end{tabular}

${ }^{1}$ Notes: OWO, overweight or obesity; SD, standard deviation; non-smoking, non-smoker (nonsmoking smoke).

${ }^{2}$ nonOWO, overweight and obesity are defined as maternal $\mathrm{BMI}<25 \mathrm{~kg} / \mathrm{m} 2,25-29: 9 \mathrm{~kg} / \mathrm{m} 2$, and $\mathrm{BMI}>30 \mathrm{~kg} / \mathrm{m} 2$, respectively.

${ }^{3}$ Preterm is defined as gestational age $<37$ weeks.

Table 3. The individual and joint associations of MOWO and maternal self-reported smoking during pregnancy (non-smoking, quit smoking, continuous) on child's OWO status during childhood (last BMI measurements) in overall samples ${ }^{1}$.

\begin{tabular}{|c|c|c|c|c|c|c|}
\hline $\begin{array}{c}\text { Maternal OWO } \\
\text { status }\end{array}$ & Smoking status & $\mathrm{N}$ & $n_{1}$ & OR $(95 \% \mathrm{CI})$ & $n_{2}$ & OR (95\%CI) \\
\hline \multicolumn{7}{|c|}{ Individual associations } \\
\hline Non OWO & & 1109 & 176 & Referent & 201 & Referent \\
\hline \multirow[t]{4}{*}{ OWO } & & 1242 & 224 & $1.61(1.27-2.03)$ & 461 & $3.02(2.46-3.71)$ \\
\hline & Non smoking & 1930 & 331 & Referent & 527 & Referent \\
\hline & Quit smoking & 188 & 27 & $0.90(0.57-1.41)$ & 61 & $1.00(0.88-1.75)$ \\
\hline & Continuous smoking & 233 & 42 & $1.21(0.81-1.80)$ & 74 & $1.28(0.92-1.79)$ \\
\hline \multicolumn{7}{|l|}{ Joint associations } \\
\hline \multirow{3}{*}{ Non OWO } & Non smoking & 927 & 144 & Referent & 165 & Referent \\
\hline & Quit smoking & 75 & 8 & $0.68(0.32-1.48)$ & 15 & $1.03(0.56-1.90)$ \\
\hline & Continuous smoking & 107 & 24 & $1.73(1.01-2.97)$ & 21 & $1.29(0.75-2.25)$ \\
\hline \multirow{3}{*}{ OWO } & Non smoking & 1003 & 187 & $1.70(1.31-2.20)$ & 362 & $2.98(2.37-3.75)$ \\
\hline & Quit smoking & 113 & 19 & $1.68(0.95-2.96)$ & 46 & $3.63(2.32-5.68)$ \\
\hline & Continous smoking & 126 & 18 & $1.45(0.81-2.58)$ & 53 & $3.66(2.37-5.67)$ \\
\hline \multicolumn{3}{|c|}{$\mathrm{p}$ for interaction (OWO*smoking) } & \multicolumn{2}{|r|}{0.453} & \multicolumn{2}{|r|}{0.664} \\
\hline
\end{tabular}

${ }^{1}$ Models were adjusted for maternal age at delivery, race, education, parity, perceived stress during pregnancy, child age, breastfeeding, gestational or preexisting diabetes. N: number of children (total $=2351) . n_{1}$ : Number of children with overweight. $n_{2}$ : Number of children with obese.

Table 4. The individual and joint associations of MOWO and maternal plasma cotinine and hydroxycotinine levels at delivery on child's OWO status during childhood (last BMI measurements) in samples with maternal cotinine ${ }^{1}$.

\begin{tabular}{|l|l|l|l|l|l|l|}
\hline $\begin{array}{l}\text { Maternal OWO } \\
\text { status }\end{array}$ & $\begin{array}{l}\text { Biomarkers of ciga- } \\
\text { rette smoking }\end{array}$ & $\mathrm{N}$ & $n_{1}$ & $\mathrm{OR}(95 \% \mathrm{CI})$ & $n_{2}(\%)$ & $\mathrm{OR}(95 \% \mathrm{CI})$ \\
\hline Individual associations
\end{tabular}




\begin{tabular}{|c|c|c|c|c|c|c|}
\hline nonOWO & & $609(0.46)$ & $91(0.15)$ & Referent & $117(0.19)$ & Referent \\
\hline \multirow[t]{9}{*}{ OWO } & & $706(0.54)$ & $117(0.17)$ & $1.53(1.11-2.10)$ & $264(0.374)$ & $2.70(2.06-3.54)$ \\
\hline & Cotinine Q1 & $331(0.25)$ & $57(0.17)$ & Referent & $85(0.26)$ & Referent \\
\hline & Cotinine Q2 & $328(0.25)$ & $44(0.13)$ & $0.73(0.46-1.14)$ & $93(0.284)$ & $1.02(0.71-1.47)$ \\
\hline & Cotinine Q3 & $327(0.25)$ & $46(0.14)$ & $0.81(0.52-1.26)$ & $95(0.291)$ & $1.08(0.75-1.55)$ \\
\hline & Cotinine Q4 & $329(0.25)$ & $61(0.19)$ & $1.37(0.87-2.15)$ & $108(0.328)$ & $1.51(1.03-2.21)$ \\
\hline & Hydroxycotinine Q1 & $576(0.44)$ & $96(0.17)$ & Referent & $159(0.28)$ & Referent \\
\hline & Hydroxycotinine Q2 & $96(0.07)$ & $14(0.15)$ & $0.86(0.46-1.63)$ & $24(0.250)$ & $0.85(0.50-1.42)$ \\
\hline & Hydroxycotinine Q3 & $314(0.24)$ & $44(0.14)$ & $0.84(0.56-1.25)$ & $94(0.299)$ & $1.05(0.77-1.45)$ \\
\hline & Hydroxycotinine Q4 & $329(0.25)$ & $54(0.16)$ & $1.08(0.73-1.62)$ & $104(0.316)$ & $1.23(0.89-1.70)$ \\
\hline \multicolumn{7}{|l|}{ Joint associations } \\
\hline nonOWO & Cotinine Q1-3 & $468(0.36)$ & $65(0.14)$ & Referent & $87(0.19)$ & Referent \\
\hline nonOWO & Cotinine Q4 & $141(0.11)$ & $26(0.18)$ & $1.63(0.95-2.79)$ & $30(0.213)$ & $1.34(0.82-2.20)$ \\
\hline OWO & Cotinine Q1-3 & $518(0.39)$ & $82(0.16)$ & $1.52(1.04-2.21)$ & $186(0.359)$ & $2.63(1.92-3.59)$ \\
\hline OWO & Cotinine Q4 & $188(0.14)$ & $35(0.19)$ & $2.44(1.47-4.05)$ & $78(0.415)$ & $3.78(2.49-5.75)$ \\
\hline nonOWO & Hydroxycotinine Q1- & $465(0.35)$ & $66(0.14)$ & Referent & $83(0.18)$ & Referent \\
\hline nonOWO & Hydroxycotinine Q4 & $144(0.11)$ & $25(0.17)$ & $1.43(0.84-2.45)$ & $34(0.236)$ & $1.56(0.97-2.52)$ \\
\hline OWO & $3 y$ & $521(0.40)$ & $88(0.17)$ & $1.69(1.17-2.45)$ & $194(0.372)$ & $3.02(2.20-4.14)$ \\
\hline OWO & Hydroxycotinine Q4 & $185(0.14)$ & $29(0.16)$ & $1.59(0.95-2.68)$ & $70(0.378)$ & $3.03(2.00-4.59)$ \\
\hline nonOWO & Sum Q1-3 & $468(0.36)$ & $67(0.14)$ & Referent & $83(0.18)$ & Referent \\
\hline nonOWO & Sum Q4 & $141(0.11)$ & $24(0.17)$ & $1.49(0.86-2.57)$ & $34(0.241)$ & $1.63(1.01-2.65)$ \\
\hline OWO & Sum Q1-3 & $518(0.39)$ & $79(0.15)$ & $1.46(1.00-2.12)$ & $194(0.375)$ & $2.96(2.15-4.06)$ \\
\hline OWO & Sum Q4 & $188(0.14)$ & $38(0.20)$ & $2.40(1.47-3.92)$ & $70(0.372)$ & 3.34(2.19-5.08) \\
\hline \multicolumn{7}{|c|}{$\mathrm{p}$ for interaction (smoking ${ }^{*} \mathrm{SGA}$ ) } \\
\hline $\begin{array}{l}\text { Continuous smok- } \\
\text { ing }\end{array}$ & SGA & & 0.091 & & 0.569 & \\
\hline Non smoking & nonSGA & & 0.637 & & 0.786 & \\
\hline Non smoking & SGA & & 0.867 & & 0.04 & \\
\hline Quit smoking & nonSGA & & 0.898 & & 0.981 & \\
\hline Quit smoking & SGA & & 0.726 & & 0.129 & \\
\hline
\end{tabular}

${ }^{1}$ Notes: sum, sum of cotinine and hydroxycotinine. Models were adjusted for maternal age at delivery, race, education, parity, perceived stress during pregnancy, child age, breastfeeding, gestational or preexisting diabetes. N: number of children (total $=1315$ ). $n_{1}$ : Number of children with overweight. $n_{2}$ : Number of children with obese.

Table 5. The individual and joint associations of MOWO and child cord blood cotinine and hydroxycotinine levels at delivery on child's OWO status during childhood (last BMI measurements) in samples with cord cotinine ${ }^{1}$.

\begin{tabular}{|l|l|l|l|l|l|l|}
\hline $\begin{array}{l}\text { Maternal OWO } \\
\text { status }\end{array}$ & $\begin{array}{l}\text { Cord biomarkers of } \\
\text { cigarette smoking }\end{array}$ & $\mathrm{N}$ & $n_{1}(\%)$ & $\mathrm{OR}(95 \% \mathrm{CI})$ & $n_{2}(\%)$ & $\mathrm{OR}(95 \% \mathrm{CI})$ \\
\hline
\end{tabular}




\begin{tabular}{|c|c|c|c|c|c|c|}
\hline \multicolumn{7}{|c|}{ Individual associations } \\
\hline nonOWO & & $421(0.47)$ & $67(0.16)$ & Referent & $83(0.20)$ & Referent \\
\hline \multirow[t]{9}{*}{ OWO } & & $472(0.53)$ & $93(0.20)$ & $1.85(1.27-2.70)$ & $173(0.367)$ & $2.74(1.97-3.82)$ \\
\hline & Cotinine Q1 & $239(0.27)$ & $41(0.17)$ & Referent & $70(0.29)$ & Referent \\
\hline & Cotinine Q2 & $208(0.23)$ & $48(0.23)$ & $1.31(0.80-2.15)$ & $45(0.216)$ & $0.72(0.45-1.14)$ \\
\hline & Cotinine Q3 & $223(0.25)$ & $32(0.14)$ & $0.81(0.47-1.38)$ & $64(0.287)$ & $0.91(0.59-1.40)$ \\
\hline & Cotinine Q4 & $223(0.25)$ & $39(0.17)$ & $1.26(0.73-2.18)$ & $77(0.345)$ & $1.28(0.82-2.01)$ \\
\hline & Hydroxycotinine Q1 & $376(0.42)$ & $64(0.17)$ & Referent & $99(0.26)$ & Referent \\
\hline & Hydroxycotinine Q2 & $71(0.08)$ & $15(0.21)$ & $1.28(0.66-2.51)$ & $18(0.254)$ & $0.99(0.53-1.85)$ \\
\hline & Hydroxycotinine Q3 & $223(0.25)$ & $36(0.16)$ & $1.01(0.63-1.62)$ & $63(0.283)$ & $1.14(0.77-1.69)$ \\
\hline & Hydroxycotinine Q4 & $223(0.25)$ & $45(0.20)$ & $1.66(1.03-2.66)$ & $76(0.341)$ & $1.57(1.05-2.36)$ \\
\hline \multicolumn{7}{|c|}{ Joint associations } \\
\hline nonOWO & Cotinine Q1-3 & $325(0.36)$ & $47(0.14)$ & Referent & $59(0.18)$ & Referent \\
\hline nonOWO & Cotinine Q4 & $96(0.11)$ & $20(0.21)$ & 2.07(1.09-3.94) & $24(0.250)$ & $1.71(0.94-3.10)$ \\
\hline OWO & Cotinine Q1-3 & $345(0.39)$ & $74(0.21)$ & $2.34(1.52-3.61)$ & $120(0.348)$ & $2.97(2.02-4.37)$ \\
\hline OWO & Cotinine Q4 & $127(0.14)$ & $19(0.15)$ & $1.77(0.93-3.37)$ & $53(0.417)$ & $3.49(2.10-5.79)$ \\
\hline nonOWO & Hydroxycotinine Q1-3 & $328(0.37)$ & $47(0.14)$ & Referent & $61(0.19)$ & Referent \\
\hline nonOWO & Hydroxycotinine Q4 & $93(0.10)$ & $20(0.22)$ & $2.13(1.13-4.03)$ & $22(0.237)$ & $1.52(0.83-2.78)$ \\
\hline OWO & Hydroxycotinine Q1-3 & $342(0.38)$ & $68(0.20)$ & $2.10(1.36-3.26)$ & $119(0.348)$ & $2.79(1.90-4.09)$ \\
\hline OWO & Hydroxycotinine Q4 & $130(0.15)$ & $25(0.19)$ & $2.57(1.41-4.68)$ & $54(0.415)$ & $3.70(2.24-6.11)$ \\
\hline nonOWO & Sum Q1-3 & $323(0.36)$ & $48(0.15)$ & Referent & $58(0.18)$ & Referent \\
\hline nonOWO & Sum Q4 & $98(0.11)$ & $19(0.19)$ & $1.77(0.94-3.36)$ & $25(0.255)$ & $1.71(0.96-3.06)$ \\
\hline OWO & Sum Q1-3 & $347(0.39)$ & $74(0.21)$ & $2.22(1.44-3.42)$ & $119(0.343)$ & $2.93(1.99-4.31)$ \\
\hline OWO & Sum Q4 & $125(0.14)$ & $19(0.15)$ & $1.80(0.94-3.43)$ & $54(0.432)$ & $3.74(2.25-6.23)$ \\
\hline \multicolumn{7}{|c|}{$\mathrm{p}$ for interaction (smoking $*$ SGA) } \\
\hline $\begin{array}{l}\text { con- } \\
\text { tinous_smoking }\end{array}$ & SGA & & 0.388 & & 0.357 & \\
\hline Non smoking & nonSGA & & 0.431 & & 0.764 & \\
\hline Non smoking & SGA & & 0.395 & & 0.008 & \\
\hline Quit smoking & nonSGA & & 0.377 & & 0.881 & \\
\hline Quit smoking & SGA & & 0.795 & & 0.368 & \\
\hline
\end{tabular}

${ }^{1}$ Notes: sum, sum of cotinine and hydroxycotinine. Models were adjusted for maternal age at delivery, race, education, parity, perceived stress during pregnancy, child age, breastfeeding, gestational or preexisting diabetes. N: number of children (total $=893) . \quad n_{1}$ : Number of children with overweight. $n_{2}$ : Number of children with obese.

\section{Discussion}

Most previous studies assessed in utero exposure to cigarette smoking using maternal self-reported data which might introduce information bias due to recall bias, single-assessment bias, underreporting, and deliberate misreporting 17-19,28,29. Biomarkers such as cotinine levels in maternal urine ${ }^{21,22}$, maternal serum ${ }^{20}$ may or may not necessarily reflect fetal exposure. Maternal smoking metabolites such as cotinine and hydroxycotinine could potentially cross the placenta and accumulate in fetal tissues ${ }^{27}$. In this study, we examined in utero exposure to cigarette smoking using cotinine and its metabolite hydroxycotinine in cord blood, in addition to self-report or biomarkers in maternal plasma. As shown in 
Supplementary Figure 3, the paired correlation among cotinine, hydroxycotinine, and the sum of both was high within maternal and cord plasma, respectively. However, they were poorly correlated between maternal and cord measurements, which could be due to several possibilities: different half-life of cotinine and hydroxycotinine metabolism and elimination rate between maternal and fetal compartment; cord blood was obtained at birth, while maternal blood was obtained within 2-3 days after delivery. Despite the lack of correlation between maternal and cord plasma nicotine metabolites, it is assuring that both maternal and cord plasma nicotine metabolites had a high concordance with maternal self-reported smoking status. Our findings imply that future studies can use either maternal or cord blood sample to detect in utero cigarette smoking exposure. In addition, findings from this study opened the door for further investigation into maternal and fetal metabolism of nicotine and transplacental passage of nicotine metabolites, which may serve as targets for developing pharmaceutical interventions.

This is the first study to simultaneously examine three types of measures of in utero cigarette smoking exposure in relation to childhood OWO: maternal self-reported smoking and measures of maternal and cord plasma biomarkers (cotinine, hydroxycotinine). A recent study applied a similar way of combining information from maternal smoking and cord cotinine to assess the effect of smoking on child celiac disease, another type of childhood disease associated with intrauterine smoking exposure ${ }^{30}$, which served as one of the rationales to conduct similar analyses for child OWO. In this study, regardless of methods of exposure assessment, we found consistent evidence that in utero exposure to cigarette smoking appears to be an obesogen - additively with maternal OWO - that increased child long-term risk of OWO up to 18 years of age. Such association was consistently demonstrated by three different measures of exposure, and the biomarkers allowed us to assess the dose-response relationships and improve the association estimates.

Consistently, previous studies have also shown that in utero exposure to cigarette smoking was associated with higher risk of child $\mathrm{OWO}^{31}$. The biological mechanisms underlying the association are not completely clear. One possibility is fetal re-programming in response to the disruption by environmental insults such as cigarette smoking exposure in order to adapt to the environmental changes. Many experimental studies highlighted the phenotypic consequences of fetal-placental changes that predispose to obesity, diabetes, hypertension, and cardiovascular disease in adulthood, often known as fetal origins of adult disease, proposed by Dr. David Barker ${ }^{32-35}$. These changes in phenotype can become permanent in adulthood and give rise to metabolic memory ${ }^{36}$, fetal primed ${ }^{37}$, and developmental plasticity ${ }^{38}$. Besides, researchers have also found evidence of maternal smoking associated with DNA methylation ${ }^{39}$, an epigenetic modification that can modulate gene expression and carry inheritable information capable of shaping phenotypes and causing diseases ${ }^{40}$.

A limitation of this study is that postnatal smoking exposures were not considered. In addition, biomarkers were only measured at one time point. Measuring biomarkers longitudinally may reveal the association between cotinine/hydroxycotinine and child OWO with more granularity at different life stages. It is also worth exploring in the future what other biomarkers are associated with child OWO. Taking these biomarkers together, we may be able to predict childhood obesity at an earlier life stage and preemptive interventions that can be taken to prevent the childhood obesity.

\section{Conclusions}

This study has made following new contributions to the field. First, this is the first large prospective birth cohort study of US high-risk minority mother-child dyads, in which we characterized fetal in utero exposure to cigarette smoking in three ways: maternal self-reported smoking status, and two nicotine biomarkers (i.e., cotinine, hydroxycotinine) measured in both maternal and cord plasma samples obtained at birth. Second, we examined the association of each of the three sources of exposure measures with child long-term risk of OWO. In this US minority birth cohort, we found that in utero cigarette 
smoking exposure defined by self-report, maternal or cord metabolites were all consistently associated with increased child long-term risk of OWO. Adding maternal and cord plasma biomarker information slightly improved the prediction accuracy of long-term child OWO risks, compared to self-report alone.

This study has multiple strengths. First, we used data from a large perspective cohort that is rich in low-income, minority populations; this is a high-risk group that has been under-represented in previous research. Second, we assessed in utero cigarette smoking exposure using self-reported data as well as biomarkers in maternal and cord plasma (i.e., direct evidence of fetal exposure). Third, our longitudinal design and robust findings further underscored the role of smoking as an obesogen by simultaneously modeling its metabolites in maternal and cord plasma in relation to child OWO. Our findings provided a strong argument for smoking cessation before pregnancy and also highlighted the need for public health intervention strategies to focus on maternal smoking - as a highly modifiable target that may alleviate the increasing obesity burden in the U.S. and globally.

Supplementary Materials: We have a supplementary file for supplementary tables and figures.

Author Contributions: Conceptualization, XW, WH, YJ; methodology, WH, YJ; formal analysis, $\mathrm{WH}$; data curation, $\mathrm{YJ}, \mathrm{WH}, \mathrm{XH}, \mathrm{GW}$; writing - original draft preparation, $\mathrm{WH}$; writing - review and editing, XW, MZ, LL, SS, HJ. All authors discussed, reviewed and approved the manuscript.

Funding: The Boston Birth Cohort (the parent study) is funded by the Maternal and Child Health Bureau (UJ2MC31074) and the National Institutes of Health (R01HD086013, R01HD041702, R01HD098232, R01ES031272). Dr. Hou is supported by the National Institutes of Health (K99HG011468). Mr. Zhang is supported by the American Heart Association (Award Number: 827990). The content is solely the responsibility of the authors and does not necessarily represent the official views of the funding agencies.

Institutional Review Board Statement: The Boston Birth Cohort has received initial and continuation approval by the institutional review board (IRB) of the Boston Medical Center and Johns Hopkins University Bloomberg School of Public Health (IRB\#: 3966/CR56).

Informed Consent Statement: Informed consent was obtained from all subjects involved in the study.

Data Availability Statement: Data and related materials available upon reasonable request to the corresponding author after the IRB review and approval.

Conflicts of Interest: The authors declare no conflict of interest. The funders had no role in the design of the study; in the collection, analyses, or interpretation of data; in the writing of the manuscript, or in the decision to publish the results.

\section{References}

1. Skinner, A. C., Ravanbakht, S. N., Skelton, J. A., Perrin, E. M. \& Armstrong, S. C. Prevalence of Obesity and Severe Obesity in US Children, 1999-2016. Pediatrics 141, doi:10.1542/peds.2017-3459 (2018).

2. Han, J. C., Lawlor, D. A. \& Kimm, S. Y. Childhood obesity. Lancet 375, 1737-1748 (2010).

3. Worldwide trends in body-mass index, underweight, overweight, and obesity from 1975 to 2016: a pooled analysis of 2416 population-based measurement studies in 128.9 million children, adolescents, and adults. Lancet 390, 2627-2642 (2017).

4. Singh, A. S., Mulder, C., Twisk, J. W., van Mechelen, W. \& Chinapaw, M. J. Tracking of childhood overweight into adulthood: a systematic review of the literature. Obes $\operatorname{Rev} 9,474-488$ (2008).

5. Park, M. H., Falconer, C., Viner, R. M. \& Kinra, S. The impact of childhood obesity on morbidity and mortality in adulthood: a systematic review. Obes Rev 13, 985-1000 (2012).

6. Quek, Y. H., Tam, W. W. S., Zhang, M. W. B. \& Ho, R. C. M. Exploring the association between childhood and adolescent obesity and depression: a meta-analysis. Obes Rev 18, $742-754$ (2017).

7. Edwards, M. in Handbook of Famine, Starvation, and Nutrient Deprivation: From Biology to Policy (eds Victor Preedy \& Vinood B. Patel) 1-21 (Springer International Publishing, 2017).

8. Braun, J. M. et al. Gestational perfluoroalkyl substance exposure and body mass index trajectories over the first 12 years of life. Int J Obes (Lond), doi:10.1038/s41366-020-00717-x (2020).

9. Mao, G. et al. Individual and Joint Effects of Early-Life Ambient Exposure and Maternal Prepregnancy Obesity on Childhood Overweight or Obesity. Environ Health Perspect 125, 067005-067005, doi:10.1289/EHP261 (2017).

10. Wang, G. et al. Association Between Maternal Exposure to Lead, Maternal Folate Status, and Intergenerational Risk of Childhood Overweight and Obesity. JAMA Netw Open 2, e1912343, doi:10.1001/jamanetworkopen.2019.12343 (2019). 
11. Zhang, M. et al. Association of prenatal antibiotics with measures of infant adiposity and the gut microbiome. Ann Clin Microbiol Antimicrob 18, 18, doi:10.1186/s12941-019-0318-9 (2019).

12. $\mathrm{Xu}, \mathrm{R}$. et al. DNA methylation mediates the effect of maternal smoking on offspring birthweight: a birth cohort study of multiethnic US mother-newborn pairs. Clin Epigenetics 13, 47, doi:10.1186/s13148-021-01032-6 (2021).

13. Gorog, K. et al. Maternal smoking during pregnancy and childhood obesity: results from the CESAR Study. Matern Child Health J 15, 985-992 (2011).

14. von Kries, R., Bolte, G., Baghi, L. \& Toschke, A. M. Parental smoking and childhood obesity--is maternal smoking in pregnancy the critical exposure? Int J Epidemiol 37, 210-216 (2008).

15. Neovius, K., Rasmussen, F., Sundström, J. \& Neovius, M. Forecast of future premature mortality as a result of trends in obesity and smoking: nationwide cohort simulation study. Eur J Epidemiol 25, 703-709 (2010).

16. Durmus, B. et al. Parental smoking during pregnancy, early growth, and risk of obesity in preschool children: the Generation R Study. Am J Clin Nutr 94, 164-171 (2011).

17. Oken, E., Levitan, E. B. \& Gillman, M. W. Maternal smoking during pregnancy and child overweight: systematic review and meta-analysis. Int J Obes (Lond) 32, 201-210 (2008)

18. Oken, E., Huh, S. Y., Taveras, E. M., Rich-Edwards, J. W. \& Gillman, M. W. Associations of maternal prenatal smoking with child adiposity and blood pressure. Obes Res 13, 2021-2028 (2005).

19. Bakker, R. et al. Assessment of maternal smoking status during pregnancy and the associations with neonatal outcomes. Nicotine Tob Res 13, 1250-1256(2011).

20. Goudarzi, H. et al. Contrasting associations of maternal smoking and pre-pregnancy BMI with wheeze and eczema in children. Sci Total Environ 639, 1601-1609 (2018).

21. Wang, X., Tager, I. B., Van Vunakis, H., Speizer, F. E. \& Hanrahan, J. P. Maternal smoking during pregnancy, urine cotinine concentrations, and birth outcomes. A prospective cohort study. Int J Epidemiol 26, 978-988 (1997).

22. Vrijheid, M. et al. Early-Life Environmental Exposures and Childhood Obesity: An Exposome-Wide Approach. Environ Health Perspect 128, 67009, doi:10.1289/ehp5975 (2020).

23. Jauniaux, E., Gulbis, B., Acharya, G., Thiry, P. \& Rodeck, C. Maternal tobacco exposure and cotinine levels in fetal fluids in the first half of pregnancy. Obstet Gynecol 93, 25-29 (1999).

24. Hukkanen, J., Jacob, P., 3rd \& Benowitz, N. L. Metabolism and disposition kinetics of nicotine. Pharmacol Rev 57, 79-115 (2005).

25. Jarvis, M. J., Russell, M. A., Benowitz, N. L. \& Feyerabend, C. Elimination of cotinine from body fluids: implications for noninvasive measurement of tobacco smoke exposure. Am J Public Health 78, 696-698 (1988).

26. Benowitz, N. L. \& Jacob, P., 3rd. Trans-3'-hydroxycotinine: disposition kinetics, effects and plasma levels during cigarette smoking. Br J Clin Pharmacol 51, 53-59 (2001).

27. Machado Jde, B., Chatkin, J. M., Zimmer, A. R., Goulart, A. P. \& Thiesen, F. V. Cotinine and polycyclic aromatic hydrocarbons levels in the amniotic fluid and fetal cord at birth and in the urine from pregnant smokers. PLoS One 9, e116293, doi:10.1371/journal.pone.0116293 (2014).

28. Dukic, V. M., Niessner, M., Pickett, K. E., Benowitz, N. L. \& Wakschlag, L. S. Calibrating self-reported measures of maternal smoking in pregnancy via bioassays using a Monte Carlo approach. Int J Environ Res Public Health 6, 1744-1759 (2009).

29. Shipton, D. et al. Reliability of self reported smoking status by pregnant women for estimating smoking prevalence: a retrospective, cross sectional study. BMJ 339, b4347, doi:10.1136/bmj.b4347 (2009).

30. Mårild, K. et al. Smoking in pregnancy, cord blood cotinine and risk of celiac disease diagnosis in offspring. Eur J Epidemiol 34, 637-649 (2019).

31. Riedel, C. et al. Parental smoking and childhood obesity: higher effect estimates for maternal smoking in pregnancy compared with paternal smoking--a meta-analysis. Int J Epidemiol 43, 1593-1606 (2014).

32. Gluckman, P. D. \& Hanson, M. A. Living with the past: evolution, development, and patterns of disease. Science 305, 1733-1736 (2004).

33. Plagemann, A. 'Fetal programming' and 'functional teratogenesis': on epigenetic mechanisms and prevention of perinatally acquired lasting health risks. J Perinat Med 32, 297-305 (2004).

34. Stocker, C. J., Arch, J. R. \& Cawthorne, M. A. Fetal origins of insulin resistance and obesity. Proc Nutr Soc 64, 143-151 (2005).

35. Barker, D. J. P. Mothers, babies, and health in later life. (Elsevier Health Sciences, 1998).

36. Yessoufou, A. \& Moutairou, K. Maternal diabetes in pregnancy: early and long-term outcomes on the offspring and the concept of "metabolic memory". Exp Diabetes Res 2011, 218598, doi:10.1155/2011/218598 (2011).

37. Bruce, K. D. \& Hanson, M. A. The developmental origins, mechanisms, and implications of metabolic syndrome. J Nutr 140, 648-652 (2010).

38. McMillen, I. C. \& Robinson, J. S. Developmental origins of the metabolic syndrome: prediction, plasticity, and programming. Physiol Rev 85, 571-633 (2005).

39. Chatterton, Z. et al. In utero exposure to maternal smoking is associated with DNA methylation alterations and reduced neuronal content in the developing fetal brain. Epigenetics Chromatin 10, 4, doi:10.1186/s13072-017-0111-y (2017).

40. Feinberg, A. P. The Key Role of Epigenetics in Human Disease Prevention and Mitigation. N Engl J Med 378, 1323-1334 (2018).

41. Wang, G. et al. Preterm birth and random plasma insulin levels at birth and in early childhood. JAMA 311, 587-596 (2014).

42. Wang, X. et al. Maternal cigarette smoking, metabolic gene polymorphism, and infant birth weight. JAMA 287, 195-202 (2002).

43. Centers for Disease Control and Prevention. Overview of the CDC Growth Charts for Use in the United States Among Children and Teens Aged 2 Years to 20 Years, <https://www.cdc.gov/nccdphp/dnpao/growthcharts/training/overview/index.html> ( 
44. Centers for Disease Control and Prevention. Defining Childhood Obesity, <https://www.cdc.gov/obesity/childhood/defining.html>

45. van Buuren, S. \& Groothuis-Oudshoorn, K. mice: Multivariate Imputation by Chained Equations in R. 2011 45, 67, doi:10.18637/jss.v045.i03 (2011).

46. Pollard, T. J., Johnson, A. E. W., Raffa, J. D. \& Mark, R. G. tableone: An open source Python package for producing summary statistics for research papers. JAMIA Open 1, 26-31 (2018).

47. Ripley, B., Venables, W. \& Ripley, M. B. Package 'nnet'. R package version 7, 3-12 (2016).

48. Tingley, D., Yamamoto, T., Hirose, K., Keele, L. \& Imai, K. mediation: R Package for Causal Mediation Analysis. 2014 59, 38 , doi:10.18637/jss.v059.i05 (2014).

49. Peterson, B. G. et al. PerformanceAnalytics: Econometric tools for performance and risk analysis. R package version 1, 107 (2014).

50 Wickham, H. ggplot2: Elegant Graphics for Data Analysis. Springer-Verlag New York. ISBN 978-3-319-24277-4, https://ggplot2.tidyverse.org (2016) 